\title{
Observation of discrete VLF emissions at low latitudes and their generation mechanism
}

\author{
Abhay Kumar Singh ${ }^{1}$ and R. P. Singh ${ }^{2}$ \\ ${ }^{1}$ Department of Physics, Maharaja College, V. K. S. University, Arrah-802301, Bihar, India \\ ${ }_{2}^{2}$ Physics Department, Banaras Hindu University, Varanasi-221005, India
}

(Received November 21, 2003; Revised November 2, 2004; Accepted November 2, 2004)

\begin{abstract}
Different type of discrete VLF emissions recorded at low-latitude ground stations Varanasi (geomag. lat., $14^{\circ} 55^{\prime} \mathrm{N}$, geomag. long., $154^{\circ} \mathrm{E}, L=1.07$ ), Jammu (geomag. lat., $22^{\circ} 26^{\prime} \mathrm{N}, L=1.17$ ) and Gulmarg (geomag. lat., $24^{\circ} 26^{\prime} \mathrm{N}$, geomag. long., $147^{\circ} 9^{\prime} \mathrm{E}, L=1.28$ ) are reported and spectral analysis of these emissions have been carried out. To explain various temporal and spectral features of these emissions, a possible generation mechanism has been presented based on the backward wave oscillator (BWO) regime of the magnetospheric cyclotron maser. On the basis of this model, we have computed various discrete emission parameters as well as some magnetospheric parameters relevant to the generation process. A comparison of the observed discrete characteristics with the proposed generation mechanism shows a good agreement with the BWO model.
\end{abstract}

Key words:

\section{Introduction}

Very low frequency (VLF) emission is a class of natural radio frequency phenomenon which is often observed in close association with whistler waves both at the Earth's surface and on satellites (Helliwell, 1965). Two different groups of magnetospheric VLF emissions: (1) unstructured continuous emissions in both time and frequency which tend to maintain a steady state like hiss, resonance bands and noise bands near the ion gyrofrequencies, (2) structured discrete emissions with a repetitive and even periodic character which tends to be transient like chorus, periodic emissions and other transient discrete emissions such as hooks, risers, fallers, pseudo whistlers, have been reported (Helliwell, 1965; Cornilleau-Wehrlin et al., 1978; Sazhin, 1982; Hattori et al., 1991; Sazhin and Hayakawa, 1992). Although it is generally accepted that their generation is connected with the electron cyclotron resonance of whistlermode waves and radiation belt electrons (Helliwell, 1967), the mechanism responsible for the origin of discrete emissions and formation of spectrum of separate elements are still a subject of active experimental and theoretical research (Smith et al., 1996; Trakhtengerts, 1999; Singh et al., 2000; Lauben et al., 2002; Singh et al., 2003; Santolik and Gurnett, 2003; Santolik et al., 2003, 2004; Singh and Patel, 2004; Singh and Ronnmark, 2004).

Unlike mid and high latitude emissions, low latitude VLF emissions have not been used much for exploring the inner magnetosphere. The main reason being the fact that the propagation characteristics of VLF emissions in the low latitude ionosphere are not properly known, since satellite and ground based results are scarce. Hence, the mechanism of

Copy right(c) The Society of Geomagnetism and Earth, Planetary and Space Sciences (SGEPSS); The Seismological Society of Japan; The Volcanological Society of Japan; The Geodetic Society of Japan; The Japanese Society for Planetary Sciences; TERRAPUB their generation, location of source and propagation from the source to the observation site are quite far from reliable understanding (Lalmani et al., 2000; Singh et al., 2000; Singh and Patel, 2004). Therefore, a better understanding of the generation mechanism of these VLF emissions observed at low latitudes would be more useful for analyzing the properties of high energy trapped electrons. During the course of our analysis of VLF data recorded at the low latitude ground stations Varanasi, Jammu and Gulmarg, we found some good examples of discrete VLF emissions which we report here with a discussion of their probable generation mechanism.

Helliwell (1967) developed a phenomenological model for the generation of discrete emissions based on the cyclotron resonance of energetic electrons with whistler mode waves, which manifest themselves as backward wave oscillators. In this model the idea of second order cyclotron resonance was first formulated and frequency spectrum of different types of discrete emissions was explained. Analytical and computational calculations supported the idea of the second order resonance and permitted a connection to be made through nonlinear currents, between the parameters of a triggered emission and those of the initial quasimonochromatic whistler wave (Nunn, 1974). Omura et al. (1991) have given a comprehensive review of the non linear theory of triggered emissions.

Trakhtengerts (1999) suggested a generation mechanism of chorus-like discrete VLF emissions based on the backward wave oscillator (BWO) regime of magnetospheric cyclotron maser (Trakhtengerts, 1995). This leads to the formation of a specific step-like deformation of the distribution function, which drastically changes all further developments of the wave-particle interactions (Trakhtengerts et al., 1996). Trakhtengerts (1995) revealed that the step-like deformation could be the cause of new generation regimes 
VARANASI
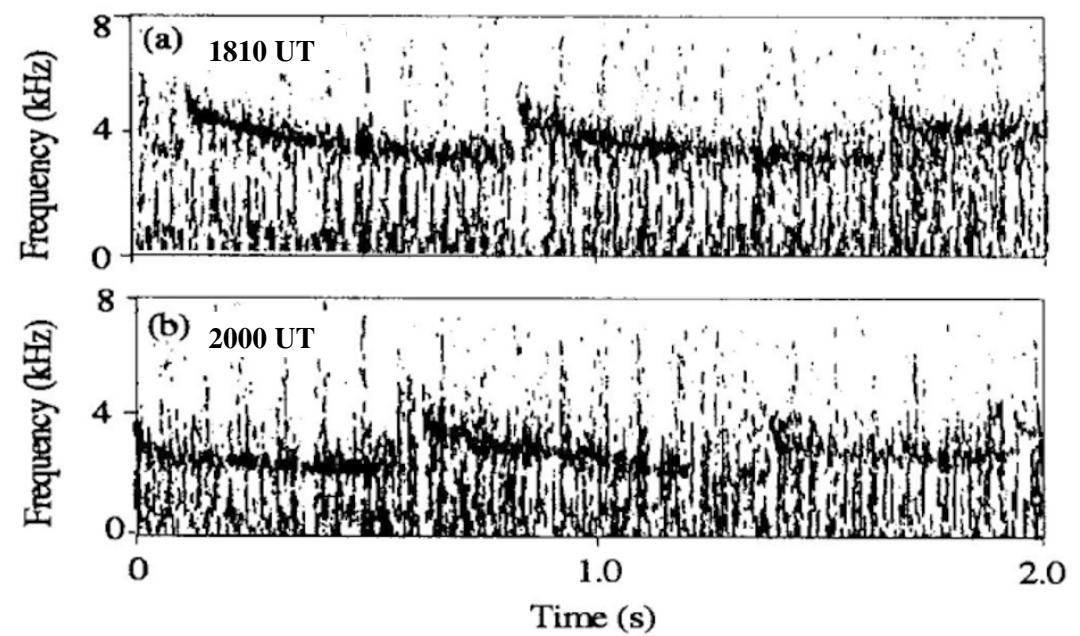

Fig. 1. Typical examples of the frequency-time spectrograms of discrete VLF emissions observed at Varanasi on 29th April, 1990.

of the cyclotron instability, which were accompanied by a succession of discrete signals. Recently, Titova et al. (2003) have given the verification of the backward wave oscillator model of VLF chorus generation using data from MAGIONS satellite.

In the present paper, first we present a detailed analysis of the discrete VLF emission events observed at Varanasi, Jammu and Gulmarg during the routine recording of whistlers. Further, a generation mechanism of these discrete VLF emissions based on the backward wave oscillator regime of magnetospheric cyclotron maser (Trakhtengerts, 1995; Trakhtengerts, 1999; Demekhov and Trakhtengerts, 2001 ) is proposed. An attempt has been made to determine various discrete emission parameters as well as magnetospheric parameters relevant to the generation process. Finally, derived and estimated parameters are compared and the results are discussed in view of the other reported work.

\section{Experimental Observation and Analysis}

At the low latitude ground stations Varanasi, Jammu and Gulmarg the wideband VLF waves were received by a Ttype antenna, suitably amplified by pre and main- amplifiers and recorded using a tape recorder. The recorded VLF data were analyzed by Sonograms and Advance VLF Data Analysis System (AVDAS). At low latitude, nights with observable VLF emissions are rather rare, and the activity is closely related to strong magnetic storms (Singh, 1993). But when it appears its occurrence rate is comparable to that of mid latitude (Singh, 1993; Singh et al., 1999). The observations of discrete VLF emissions at low latitude ground stations Varanasi, Jammu and Gulmarg are unusual in the sense that most of the reported observations are from satellites (Cornilleau-Wehrlin et al., 1978; Hattori et al., 1991; Santolik and Gurnett, 2003; Santolik et at., 2004) or from high latitudes. In this paper, the discrete VLF emissions recorded in large numbers in the night of 29th April, 1990 at Varanasi were analyzed first. Typical spectrograms of the discrete VLF emissions recorded at Varanasi are shown in Fig. 1. Figure 1(a) contains three discrete VLF emissions (falling tones) observed at 1810 UT with a background hiss band. The mean upper boundary frequency $\left(f_{U B}\right)$ for these emissions is $5.5 \mathrm{kHz}$. In Fig. 1(b) we find four discrete emissions observed at 2000 UT with mean upper boundary frequency of $5 \mathrm{kHz}$. These discrete emissions started at $1810 \mathrm{UT}$ in the frequency range of about $2.0-5.5 \mathrm{kHz}$. In course of time the upper band frequency increases to $6 \mathrm{kHz}$ and then decreases to $5 \mathrm{kHz}$ by 2000 UT. The observed discrete emissions have the following parameters: $f_{\min }=2.4$ $\mathrm{kHz}, f_{\max }=6 \mathrm{kHz}$, average $f_{U B}=5.5 \mathrm{kHz}$, average frequency sweep rate, $d f / d t=2.5 \mathrm{kHz} / \mathrm{s}$ and average duration of each discrete element, $T=0.5 \mathrm{~s}$.

In our analysis system the emissions are displayed for $2.25 \mathrm{sec}$ during one shot. We had analyzed the whole events and procured only two pieces of the emissions because it was similar everywhere. To discuss the presence of fine structures we need the variation of $d f / d t$ with time $t$. As the system is not in the working condition the fine structures present in the emissions, if any, could not be studied. We could discuss only overall $d f / d t$ slope of the elements. The other limitation is the evaluation of amplitude of the emissions recorded at different stations. Although, the antenna and receiving setup used in the observation at different stations are identical. Our analysis system does not permit us to obtain the calibrated amplitude of the events. The amplitude of the events is theoretically estimated and a direct comparison could have been made with the observed value, which may have enabled us to make some remarks about the generation mechanism, mode of propagation and ambient parameters of the medium. Under these limitations we present some useful observations recorded at low latitude Indian stations.

These emissions were recorded during the strong magnetic activity period on 29-30 April, 1990 with minimum Dst-index of $-98 \mathrm{nT}$ on 29th April. The Dst-index variation during the storm period is shown in Fig. 2, in which emission events at Varanasi are also marked. The intense discrete emissions were registered in the night of 29th April, when the magnetic activity was highest $\left(\Sigma K_{p}=32\right)$, dur- 


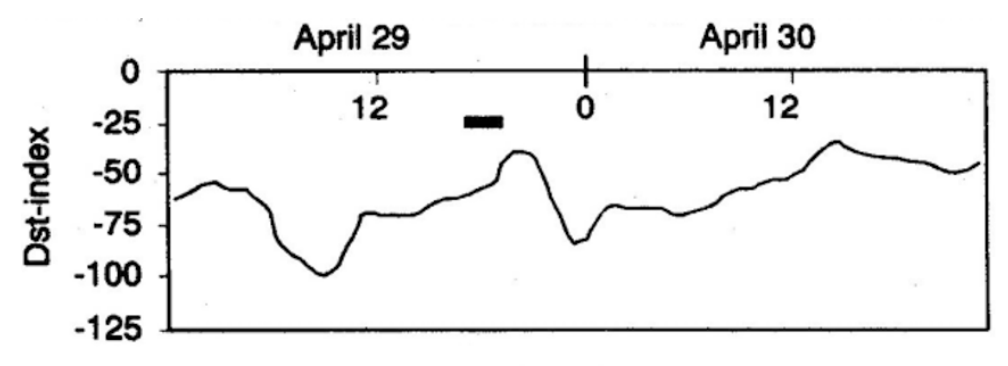

Time (UT)

Fig. 2. The Dst-index variation during the magnetic storm of 29-30 April, 1990. The duration of discrete VLF emissions is also marked.

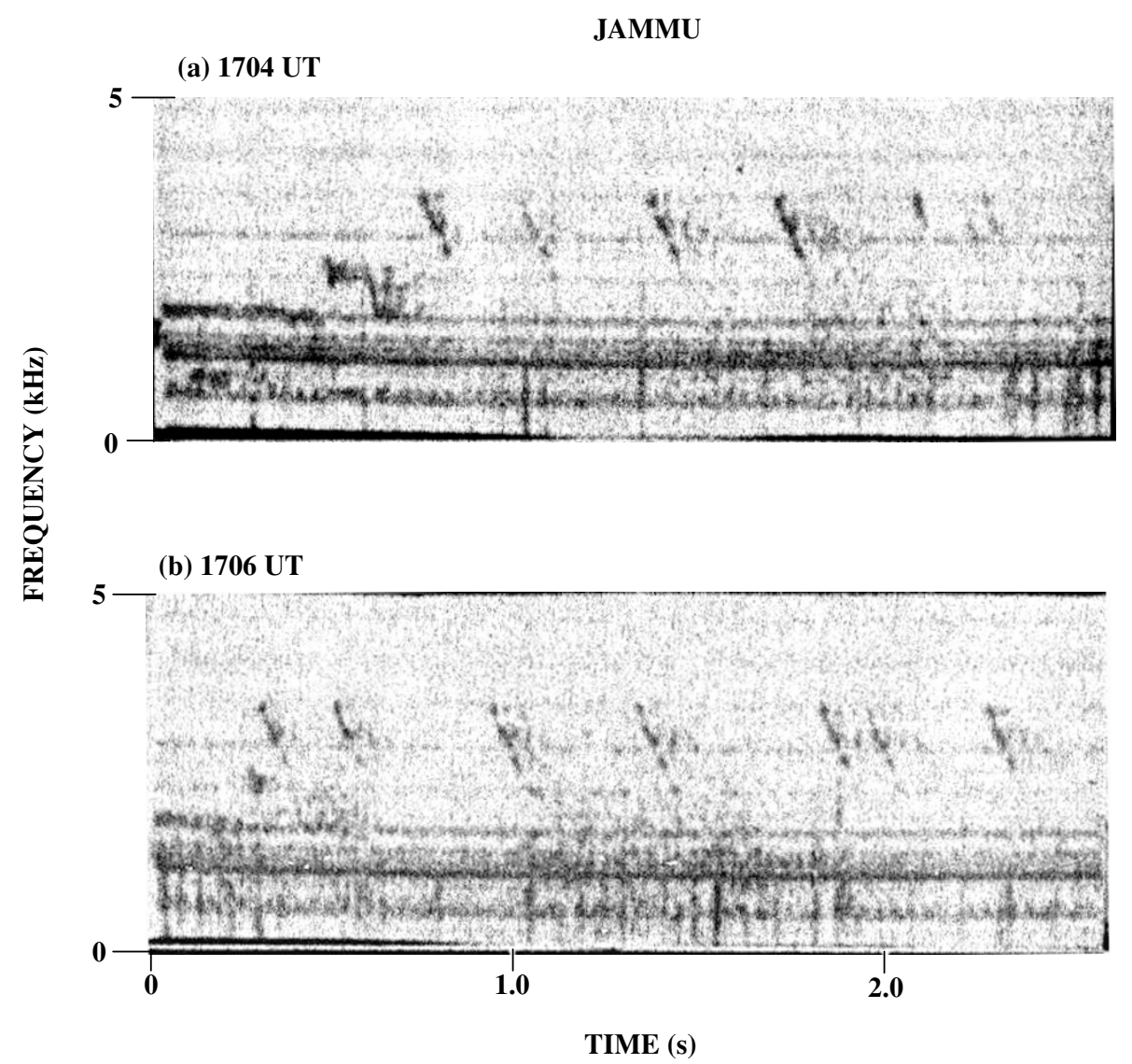

Fig. 3. Typical examples of frequency-time spectrograms of discrete VLF emissions observed at Jammu on 5th June, 1997.

ing the recovery phase of sub storm.

Secondly we have analyzed discrete VLF emissions recorded at low latitude ground station Jammu during a quiet day on the 5th June, 1997. The frequency-time spectrograms of these VLF emissions are shown in Fig. 3. On the 5th June, 1997 at station Jammu, the periodic emission activity started at around 1610 UT (2140 LT) and lasted for about one hour ending finally at 1715 UT. Figure 3(a) shows seven discrete VLF emissions, which are falling tones observed at 1704 UT. The mean $f_{U B}$ for these emissions is $3.8 \mathrm{kHz}$ with lower cut off frequency, $f_{\min }=1.8$ kHz. In Fig. 3(b) we observed six discrete elements at 1706 UT. Here, a clear periodic structure with a period of about $0.33 \mathrm{~s}$ appears. The observed mean discrete element has the following parameters: $f_{U B}=3.8 \mathrm{kHz}, f_{\min }=1.8 \mathrm{kHz}$, frequency sweep rate $d f / d t=15 \mathrm{kHz} / \mathrm{s}$ and successive repetition period $T=0.33 \mathrm{~s}$.

Typical frequency-time spectrograms of the third type of discrete VLF emissions (chorus emissions) recorded during nighttime, between 21:26 UT to 01:23 UT, on 7-8 March 1986 at low latitude ground station Gulmarg, is shown in Fig. 4 (Singh et al., 2000). The hiss is seen as an unstructured signal below about $2.5 \mathrm{kHz}$, and the discrete rising tones are chorus emissions. It is also observed from the figure that each chorus element originates from the upper edge of the underlying hiss band. In general, the chorus occurrence rate and intensity is found to be closely correlated with the intense hiss activity. These discrete chorus events started at 21:26 UT (02:56 LT) with an upper boundary frequency $f_{U B}$ of $5.8 \mathrm{kHz}$. Figure 4(a) shows chorus 


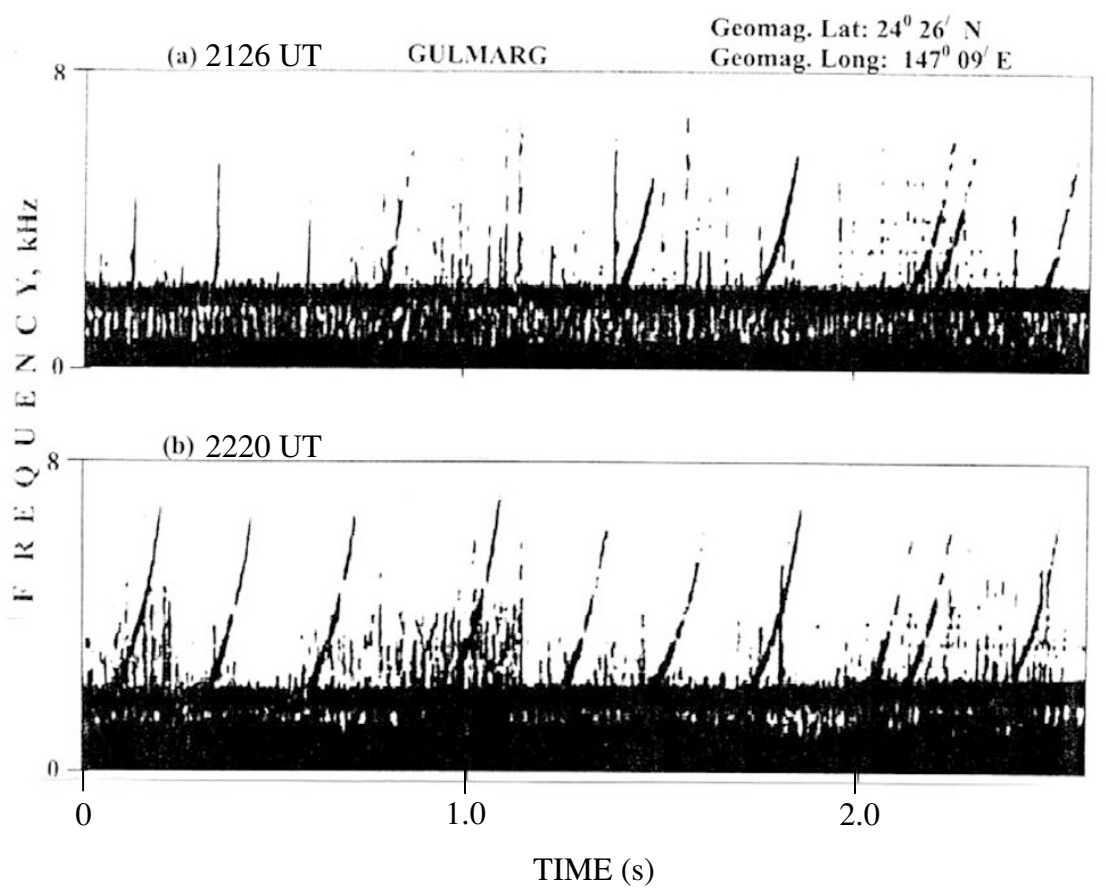

Fig. 4. Typical examples of frequency-time spectrograms of discrete chorus emissions recorded at Gulmarg on 7-8 March, 1986.
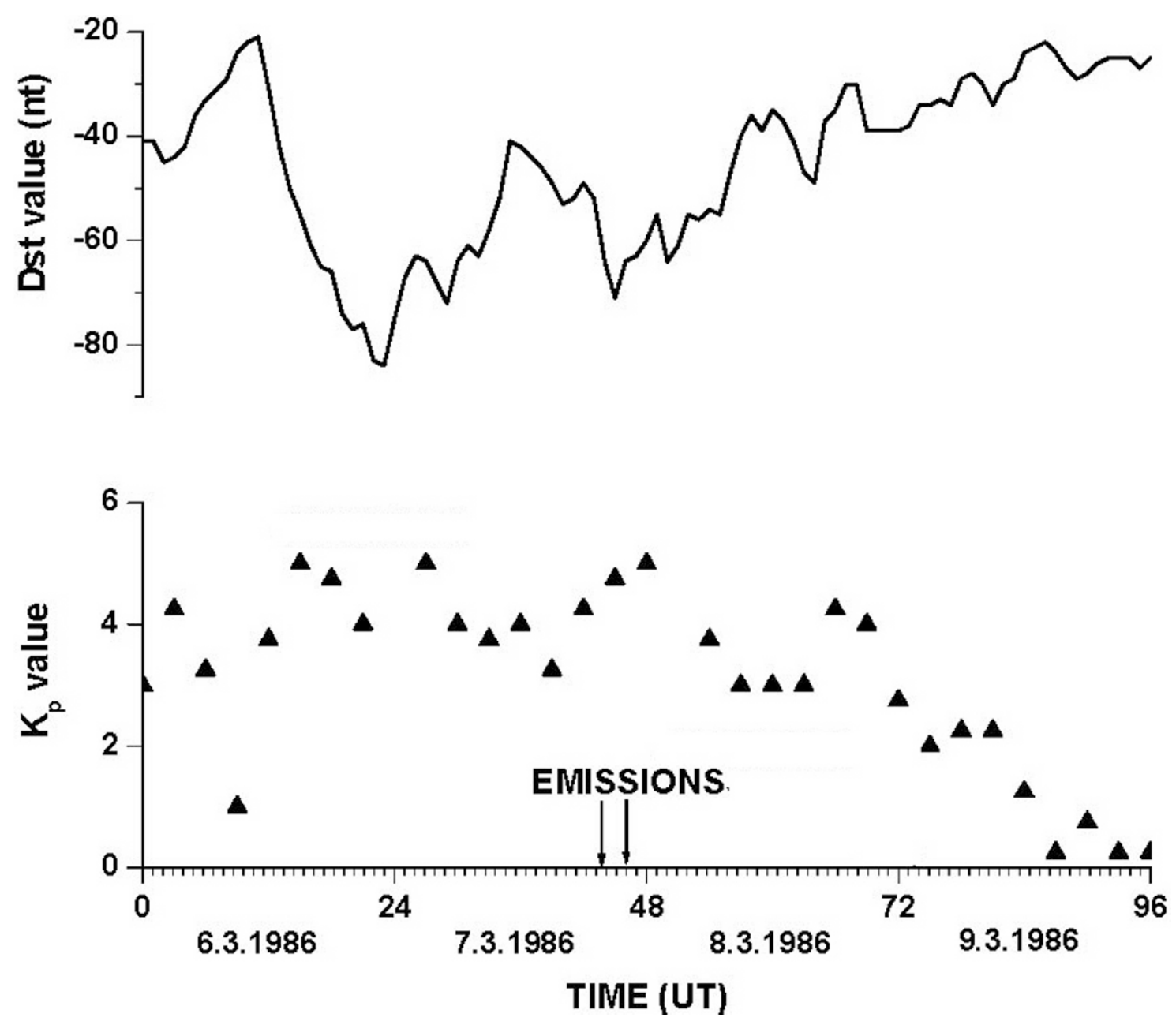

Fig. 5. The Dst-index and $k_{p}$-index variations during the magnetic storm periods of 6-9 March, 1986. The observation period of the event is also marked. 
risers observed at 21:26 UT in the frequency range 2.4-5.8 $\mathrm{kHz}$. The average spacing between the traces is about 0.28 s. Over the course of time the upper boundary frequency began to increase and it reached $7.2 \mathrm{kHz}$ by $22: 20 \mathrm{UT}$, as shown in Fig. 4(b). Here, a clear periodic structure with a period of about $0.29 \mathrm{~s}$ appears. For the next two hours the upper boundary frequency remained the same. From 00:20 UT, it began to slowly decrease and by 01:23 UT reached $4.6 \mathrm{kHz}$. The whole discrete chorus event lasted for more than $4 \mathrm{~h}$. The observed mean chorus element has the following parameters: $f_{\min }=2.4 \mathrm{kHz}, f_{U B}=6.5 \mathrm{kHz}$, frequency sweep rate $d f / d t=40 \mathrm{kHz} / \mathrm{s}$ and successive repetition pe$\operatorname{riod}, T=0.3 \mathrm{~s}$.

These discrete chorus emissions were recorded during the strong magnetic storm period of 6-9 March, 1986 with minimum Dst- index of $-84 \mathrm{nT}$ on 6 March and maximum $\Sigma K_{p}=34$ on 8 March. The Dst-index variations and $k_{p^{-}}$ index variations are shown in Fig. 5, in which the observation period of the event is also marked.

\section{Generation Mechanism}

The magnetosphere cyclotron maser (MCM) concept has been used to describe origin of different types of VLF emissions (Trakhtengerts, 1979; Bespalov and Trakhtengerts, 1986), in which separate magnetic flux tubes are considered as resonant cavities for whistler waves with conjugate ionospheres as mirrors for electromagnetic waves. Whistler wave packets propagating between mirrors amplify during interactions with trapped energetic electrons. In such a system there are different generation regimes (stationary regime with hiss generation, relaxation oscillation regime with generation of quasi-periodic emission, spike-like generation regime), which are similar to laboratory masers. However, mechanisms responsible for the origin of discrete VLF emission, temporal succession and formation of spectrum of separate elements can not be explained by the simple MCM concept.

Recently, Trakhtengerts (1995, 1999) suggested a new generation regime of MCM, the backward wave oscillator (BWO) regime discovered in electronic devices many years ago (Johnson, 1955). For a generator to operate in the BWO regime certain conditions have to be satisfied. The first condition requires that the phase velocity component along the magnetic field should be opposite to the electron motion. This is realized in the cyclotron resonance

$$
\omega-\omega_{B}=k v
$$

when $\omega<\omega_{B}$. Where, $\omega_{B}$ is the electron gyrofrequency, $\omega$ is the wave frequency, $k$ and $v$ are the components of the wave vector and electron velocity along the magnetic field. MCM operates on the same resonance and serves as a cosmic analog to laboratory cyclotron resonance masers. The second condition is the existence of a well organized electron beam with small velocity dispersion in the region of discrete emission generation. This condition poses a significant problem, since there is no obvious reason for such a beam to be formed. The solution of this problem can be related to the fact that cyclotron interaction of band-limited natural ELF/VLF noise-like emissions with energetic electrons results in formation of specific step-like features of the distribution function (Nunn and Sazhin, 1991). This steplike deformation of energetic electron distribution function ensures large growth rate $\left(\gamma_{H D}\right.$ of whistler waves and transition to the BWO regime. Trakhtengerts (1995) showed that the step-like deformation of the distribution function, caused by interactions of the natural ELF/VLF noise like emissions and energetic electrons, acts in the magnetosphere as a well organized beam in laboratory devices. The magnetospheric BWO has no fixed boundaries, and its interaction length $l$ is determined by the inhomogeneity of the geomagnetic field. According to Helliwell (1967) and Trakhtengerts $(1995,1999)$, the interaction length $l$ between the whistler waves and energetic electrons is limited by

$$
l_{0}<l<l_{m}
$$

with

$$
l_{0}=\left(\frac{L^{2} R_{E}^{2}}{k}\right)^{1 / 3}
$$

and

$$
l_{m}=\frac{2 \sqrt{2}}{3} L R_{E}\left[\left\{1+4 \frac{\left(f_{U B}-f_{\min }\right)}{f_{B}}\right\}^{1 / 2}-1\right]^{1 / 2}
$$

where, $R_{E}$ is the Earth's radius, $L$ is the McIlwain parameter and wave number $k$ is given by the whistler dispersion relation as $k=\omega^{1 / 2} \omega_{P} c^{-1} /\left(\omega_{B}-\omega\right)^{1 / 2}$, where $c$ is the velocity of light.

The BWO generation starts when the beam density exceeds some threshold value. With the increase of the beam density the BWO regime changes from a continuous regime to a periodic regime, which is accompanied by a periodic modulation of wave intensity, and then to stochastic modulation. According to Trakhtengerts (1995), the BWO threshold condition can be written as

$$
p=\frac{2 \gamma_{H D} l}{\pi\left(v v_{g}\right)^{1 / 2}} \cong 2
$$

where, $l$ is working length of the magnetospheric generator, $v_{g}$ is the group velocity of the whistler mode waves, $\gamma_{H D}$ is the hydrodynamic growth rate in the case of the distribution function with a step-like deformation. The growth rate of BWO generation with periodic modulation can be written as (Trakhtengerts, 1995):

$$
\gamma_{B W O}=\frac{2 p(p-1)}{T}
$$

where

$$
T=1.5 l\left(\frac{1}{v_{g}}+\frac{1}{v}\right)
$$

is the modulation period, which is connected with the repetition period of discrete VLF emission elements.

After the transition to the BWO regime, the dynamical spectrum of a separate discrete element is formed similar to discrete signals triggered by VLF transmitters (Nunn, 1974). According to computer simulation of triggered emissions (Nunn, 1974; Omura et al., 1991) the optimal condition for the generation of discrete emissions, corresponding 
to the maximum value of the non-linear growth rate, can be written as

$$
0.2 \leq|S| \leq 0.8
$$

where

$$
S=\frac{1}{\Omega_{t r}^{2}}\left[-\frac{3 v}{2} \frac{\partial \omega_{B}}{\partial z}-\left(1+\frac{\omega_{B}}{2 \omega}\right) \frac{d \omega}{d t}\right]
$$

and the trapping frequency $\Omega_{t r}$ is determined by the expression

$$
\Omega_{t r}=\left(k u \omega_{B} b\right)^{1 / 2}
$$

here, $b=B_{\sim} / B_{L}$ where, $B \sim$ is the whistler wave magnetic field amplitude, $B_{L}$ is the geomagnetic field and $u$ is the electron velocity component across the geomagnetic field. We can estimate the wave amplitude $B \sim$ from the relation, which is valid in the case of an absolute instability (Trakhtengerts, 1984),

$$
\frac{\Omega_{t r}}{\gamma_{B W O}} \cong \frac{32}{3 \pi}
$$

where $\gamma_{B W O}$ is determined by Eq. (4). From Eqs. (7) and (8) we can estimate the wave amplitude $B \sim$ as

$$
B_{\sim}=\left(\frac{32}{3 \pi}\right)^{2} \frac{\gamma_{B W O}^{2} B_{L}}{k u \omega_{B}} .
$$

In our case the generated wave propagates from the equator towards the increasing magnetic field, so $S<0$ (Omura et al., 1991). Putting $S=-0.5$ and substituting into Eq. (7) the value of $\Omega_{t r}$, we get the frequency sweep rate as (Trakhtengerts, 1999)

$$
\frac{d f}{d t}=1.5 \frac{\omega \gamma_{B W O}^{2}}{\omega_{H}+2 \omega}\left(1+S_{0}\right)
$$

where

$$
f=\frac{\omega}{2 \pi}, S_{0}=\left(\frac{v}{3 \gamma_{B W O}^{2}}\right)\left(\frac{\partial \omega_{B}}{\partial z}\right) .
$$

To test this theory of generation mechanism we have computed various discrete emission parameters using the above Eqs. (1)-(10) and compared them with our observations.

\section{Results and Discussions}

The observations of VLF emissions at low latitude station Varanasi, Jammu and Gulmarg show that the occurrence rate of discrete VLF emissions is low and sporadic. It is also noticed that the number of VLF emissions recorded during magnetic storm period are large. Similar occurrence rate of VLF emissions were reported by Singh et al. (2000) at low latitude ground station Gulmarg. The morphological characteristics of discrete VLF emissions at low latitudes have been studied by Lalmani et al. (2000), which reported the maximum occurrence in the month of April.

Observations of chorus events by satellites near the geomagnetic equator (Tsurutani and Smith, 1974) support the idea that the source of chorus emissions is located most probably near equatorial region. By analyzing POLAR satellite data Lauben et al. (2002) have studied various source characteristics of chorus and indicated the likely source region near the magnetic equator. Recently, Santolik et al. $(2003,2004)$ have investigated intense whistlermode chorus emissions which occurred during geomagnetic storm. These chorus emissions were measured by the four Cluster satellites below one half of the electron cyclotron frequency, at a radial distance of 4.4 Earth's radii, within a $2000 \mathrm{~km}$ long region located closed to the equator.

The outer boundary of the plasmapause has a focusing property for the discrete VLF emissions, and the maximum of chorus intensity is found to move towards lower latitudes with the increase in geomagnetic activity (Smirnova et al., 1976; Smirnova, 1984). The range of observed discrete chorus frequencies is controlled by the equatorial electron gyrofrequencies (Burtis and Helliwell, 1976). We have followed the upper boundary frequency (UBF) method developed by Smirnova (1984), to find out the location of source for the recorded discrete emission events. The upper boundary frequency of the ground based discrete VLF emissions is determined on the assumption of dipolar geomagnetic field configuration, by the half equatorial electron gyrofrequency in the generation region, irrespective of the latitude of the observation station. The $L$-value of the discrete emission source is then computed as (Smirnova, 1984)

$$
L=\left(440 / f_{U B}\right)^{1 / 3}
$$

where, $f_{U B}$ is in $\mathrm{kHz}$. Using the above method the $L$-value of the source region for the reported events of discrete VLF emissions are found to be $L_{\text {source }}=4.3,4.8$ and 4.07 for the first, the second and the third types respectively. The higher $L$-value of the source compared to that of Varanasi $(L=1.07)$, Jammu $(L=1.17)$ and Gulmarg $(L=1.28)$ shows that the wave may have propagated towards significantly lower latitudes, as expected during strong geomagnetic activity (Smirnova et al., 1976; Smirnova, 1984). The whistler mode wave propagating through the magnetosphere may shift towards lower $L$-shells (Storey, 1953). In this process $d f / d t$ also increases as the wave has to travel longer distance leading to change in dynamic spectrum (Chum et al., 2003).

To examine the generation mechanism we have computed various emission parameters using the above equations (1) to (11). For the first type of discrete VLF emissions observed at Varanasi (Fig. 1), the magnetic field at the source is about $B_{L}=3.9 \times 10^{-7} \mathrm{~T}$ and the equatorial electron gyrofrequency is $f_{B} \sim 11 \mathrm{kHz}$. From the empirical equatorial electron density profile reported by Carpenter and Anderson (1992) the electron density is $\sim 16$ electrons $\mathrm{cm}^{-3}$ and the corresponding plasma frequency is $f_{P} \sim 36$ $\mathrm{kHz}$. Considering a frequency $f=f_{B} / 3$ we find from the dispersion relation that $k \sim 0.5 \mathrm{~km}^{-1}$. From Eq. (2) the interaction length $l$ should be between $l_{0}=1150 \mathrm{~km}$ and $l_{m}=17320 \mathrm{~km}$. Using the resonance condition (1) we find that the group velocity, $v_{g} \sim 2 \omega^{2} /\left(\omega_{B} k\right) \sim 3.1 \times 10^{7}$ $\mathrm{m} / \mathrm{s}$ which is lower than the velocity of resonant electrons, $v \sim 9.2 \times 10^{7} \mathrm{~m} / \mathrm{s}$. The modulation period, which determines the repetition period of discrete elements, is found to be between $0.07 \mathrm{~s}$ and $1.1 \mathrm{~s}$. Our experimental value of $T=0.5 \mathrm{~s}$ is in this interval, and suggests that the real interaction length is around $7730 \mathrm{~km}$. Using the value of $T=0.5$ and $p=2$ in Eq. (4) we find the growth rate $\gamma_{B W O}=8 \mathrm{~s}^{-1}$. This rather low growth rate, compared to, for example, $\gamma_{B W O}=50 \mathrm{~s}^{-1}$ for $L=4$ found by Demekhov and Trakhtengerts (2001), is consistent with an 
Table 1. Observed and computed parameters of discreet VLF emissions at low latitude ground stations.

\begin{tabular}{|c|c|c|c|c|c|c|c|}
\hline S.No. & Parameters & \multicolumn{2}{|c|}{ Varanasi } & \multicolumn{2}{c|}{ Jammu } & \multicolumn{2}{c|}{ Gulmarg } \\
\hline & & Observed & Computed & Observed & Computed & Observed & Computed \\
\hline 1. & $\mathrm{~L}_{\text {source }}$ & - & 4.3 & - & 4.8 & - & 4.07 \\
\hline 2. & $\begin{array}{c}\mathrm{f}_{\mathrm{UB}} \\
(\mathrm{kHz})\end{array}$ & 5.5 & - & 3.8 & - & 5.8 & - \\
\hline 3. & $\mathrm{~T}(\mathrm{~s})$ & 0.5 & $0.07<\mathrm{T}<1.1$ & 0.33 & $0.12<\mathrm{T}<1.8$ & 0.3 & $0.06<\mathrm{T}<0.94$ \\
\hline 4. & $1(\mathrm{~km})$ & 7730 & $\begin{array}{c}1_{0}=1150 \\
1_{\mathrm{m}}=17320\end{array}$ & 3470 & $\begin{array}{c}1_{0}=1240 \\
1_{\mathrm{m}}=19050\end{array}$ & 5000 & $1_{0}=1100$ \\
\hline 5. & $\begin{array}{c}\mathrm{df} / \mathrm{dt} \\
(\mathrm{kHz} / \mathrm{s})\end{array}$ & 2.5 & 19.2 & 15.0 & 43.0 & 40 & 53.0 \\
\hline 6. & $\left.\gamma_{\mathrm{BWO}}(\mathrm{s})^{-1}\right)$ & - & 8.0 & - & 12.0 & - & 13.3 \\
\hline 7. & $\mathrm{~B} \sim(\mathrm{m} \gamma)$ & - & 0.2 & - & 0.6 & - & 0.4 \\
\hline
\end{tabular}

interaction length significantly larger than $l_{0}$. Considering $S_{0} \ll 1$, the frequency sweep rate is determined from Eq. (10) as $d f / d t=19.2 \mathrm{kHz} / \mathrm{s}$, which is some what larger than the observed value of $2.5 \mathrm{kHz} / \mathrm{s}$. Using Eq. (9), we can compute the amplitude of the discrete emission, $B \sim$ by putting $\gamma_{B W O}=8 \mathrm{~s}^{-1}$ and $B_{L}=3.9 \times 10^{-7}$ Tesla for $L=4.3$, as $B_{\sim}=0.2 \mathrm{~m} \gamma$.

For the second type of discrete VLF emissions observed at Jammu (Fig. 3) the magnetic field at the source is about $B_{L}=2.7 \times 10^{-7}$ Tesla and the equatorial electron gyrofrequency is $f_{B} \sim 7.6 \mathrm{kHz}$. From Eq. (2) the interaction length $l$ should be between $l_{0}=1240 \mathrm{~km}$ and $l_{m}=19050 \mathrm{~km}$. Putting the value of $v_{g} \sim 2.1 \times 10^{7} \mathrm{~m} / \mathrm{s}$ and $v \sim 6.3 \times 10^{7}$ $\mathrm{m} / \mathrm{s}$ in Eq. (5), the modulation period is found to be between $0.12 \mathrm{~s}$ and $1.8 \mathrm{~s}$. Our experimental value of $T=0.33 \mathrm{~s}$ is in this interval, and suggests that the real interaction length is found around $3470 \mathrm{~km}$. From Eq. (4) we found that the growth rate $\gamma_{B W O}=12 \mathrm{~s}^{-1}$. From Eq. (10) we determine the frequency sweep rate, $d f / d t=43 \mathrm{kHz} / \mathrm{s}$, which is some what larger than our observed value of $15 \mathrm{kHz} / \mathrm{s}$. Using Eq. (9), the amplitude of the discrete VLF emission is computed as $B_{\sim}=0.6 \mathrm{~m} \gamma$.

For the third type of discrete chorus emissions observed at Gulmarg (Fig. 4), the magnetic field at the source is about $B_{L}=4.6 \times 10^{-7} \mathrm{~T}$, and the equatorial electron gyrofrequency is $f_{B} \sim 13 \mathrm{kHz}$. From the Carpenter and Anderson (1992) empirical equatorial electron density profile evaluated for a plasmapause at $L=4.0-4.1$ we find the electron density to be $\sim 16$ electrons $\mathrm{cm}^{-3}$ and the corresponding plasma frequency is $f_{p} \sim 36 \mathrm{kHz}$. Considering a frequency $f=f_{B} / 3$ we find from the dispersion relation that $k \sim 0.5 \mathrm{~km}^{-1}$. From Eq. (2) the interaction length $l$ should be between $l_{o}=1100 \mathrm{~km}$ and $l_{m}=17000 \mathrm{~km}$. Using the resonance condition (1) we find that the group velocity, $v_{g} \sim 3.6 \times 10^{7} \mathrm{~m} / \mathrm{s}$ is lower than the velocity of resonant electrons, $v \sim 1.1 \times 10^{8} \mathrm{~m} / \mathrm{s}$. The modulation period, which determines the repetition period of discrete elements, is then found from Eq. (5) to be between $0.06 \mathrm{~s}$ and $0.94 \mathrm{~s}$. Our experimental values of $T=0.3 \mathrm{~s}$ is in this interval, and suggests that the real interaction length is around $5000 \mathrm{~km}$. Using the value of $T=0.3$ and $p=2$ in Eq. (4), we find the growth rate, $\gamma_{B W O}=13.33 \mathrm{~s}^{-1}$. This rather low growth rate, compared to, for example, $\gamma_{B W O}=50 \mathrm{~s}^{-1}$ for $L=4$ found by Dmekhov and Trakhtengerts (2001), is consistent with an interaction length significantly larger than $l_{o}$. Considering $S_{o} \ll 1$, the frequency sweep rate is determined from Eq. (10) as $d f / d t=53 \mathrm{kHz} / \mathrm{s}$, which is comparable to our observed value of $40 \mathrm{kHz} / \mathrm{s}$. Using Eq. (9), we can compute the amplitude of the discrete chorus emissions, $B_{\sim}=0.4 \mathrm{~m} \gamma$.

The parameters of discrete VLF emissions are comparable with those derived from BWO model (Table 1). Although the theoretical estimate for the repetition period is rather rough, but the observed period is well centered in the predicted interval. The average frequency sweep rate calculated from theory is consistent with the observations.

\section{Conclusion}

Spectral analysis of three different types of discrete VLF emissions observed at the low latitude ground stations Varanasi, Jammu and Gulmarg have been carried out and generation mechanism for various temporal and spectral features of these emissions is presented on the basis of the backward wave oscillator regime of magnetospheric cyclotron maser. On the one hand this model connects directly with the theory of triggered VLF emissions, and on the other hand it explains peculiarities of discrete emissions such as its connection with hiss, the appearance of a succession of discrete elements with small repetition frequency and amplitude of a signal. On the basis of this theory, various discrete emission parameters as well as some magnetospheric parameters taking part in the generation process are computed. These parameters are comparable to that of the observed values, which support the possibility for a wide use of discrete VLF data for the ground-based diagnostics of the state of the magnetospheric plasma during sub storm.

Further experimental and theoretical studies of discrete VLF emissions at low latitude would definitely contribute to a more detailed understanding of this phenomenon.

Acknowledgments. A. K. Singh thanks Department of Science and Technology, New Delhi, India for providing BOYSCAST Fel- 
lowship. The work is partly supported by the Department of Science and Technology, New Delhi, Govt. of India under SERC projects.

\section{References}

Bespalov, P. A. and V. Y. Trakhtengerts, The cyclotron instability of the Earth's radiation belts, in Reviews of Plasma Physics, edited by M. A. Leontovich, vol. 10, 155-292, Plenum, New York, 1986.

Burtis, W. J. and R. A. Helliwell, Magnetospheric chorus: Occurrence patterns and normalized frequency, Planet. Space Sci., 24, 1007-1024, 1976.

Carpenter, D. L. and R. R. Anderson, An ISEE/whistler model of equatorial density in the magnetosphere, J. Geophys. Res., 97, 1097-1108, 1992.

Cornilleau-Wehrlin, N., R. Gendrin, F. Lefeuvre, M. Parrot, R. Grard, and D. Jones, VLF waves observed onboard GEOS-1, Space Sci. Rev., 22, 371-382, 1978.

Chum, J., F. Jiříček, J. Šmilauer, D. Shklyar, Magion 5 observations of chorus-like emissions and their propagation features as inferred from ray-tracing simulation, Ann. Geophysicae, 21, 2293-2302, 2003.

Demekhov, A. G. and V. Y. Trakhtengerts, Theory of generation of discrete ELF/VLF emissions in the Earth's magnetosphere, Radiophys. Quant. Elec., 44(1-2), 103-116, 2001.

Hattori, K., M. Hayakawa, D. Lagoutte, M. Parrot, and F. Lefeuvre, Further evidence of triggered chorus emissions from wavelets in the hiss band, Planet. Space Sci., 39, 1465-1472, 1991.

Helliwell, R. A., Whistlers and Related Ionospheric Phenomena, Stanford Univ. Press, Stanford, USA, 1965.

Helliwell, R. A., A theory of discrete emissions from the magnetosphere, J. Geophys. Res., 72, 4773-4790, 1967.

Johnson, H. R., Backward wave oscillator, Proc. IEEE, 43, 684, 1955.

Lalmani, M. K. Babu, R. Kumar, R. Singh, and A. K. Gwal, An explanation of daytime discrete VLF emissions observed at Jammu $(L=1.17)$ and determination of magnetospheric parameters, Ind. J. Phys., 74B(2), 117-123, 2000.

Lauben, D. S., U. S. Inan, T. F. Bell, and D. A. Gurnett, Source characteristics of ELF/VLF chorus, J. Geophys. Res., 107(A12), 1429, doi: 10.1029/2000JA003019, 2002.

Nunn, D., A self-consistent theory of triggered VLF emissions, Planet. Space Sci., 22, 349-378, 1974.

Nunn, D. and S. S. Sazhin, On the generation mechanism of hiss-triggered chorus, Ann. Geophysicae, 9, 603-613, 1991.

Omura, Y., D. Nunn, H. Matsumoto, and M. J. Rycroft, A review of observational, theoretical and numerical studies of VLF triggered emissions, J. Atmos. Terr. Phys., 53, 351-368, 1991.

Santolik, O. and D. A. Gurnett, Transverse dimensions of chorus in the source region, Geophy. Res. Lett., 30(2), 1031, doi: 1029/2002GL016178, 2003.

Santolik, O., D. A. Gurnett, J. S. Pickett, and M. Parrot, CornilleauWehrlin, Spatiotemporal structure of storm-time chorus, J. Geophys. Res., 108(A7), 1278, doi:1029/2002JA009791, 2003.

Santolik, O., D. A. Gurnett, J. S. Pickett, and M. Parrot, CornilleauWehrlin, A microscopic and nanoscopic view of storm time chorus on 31 March, Geophys. Res. Lett., 31, LO2801, doi:
10.1029/2003GL018757, 2004.

Sazhin, S. S., Natural Radio Emissions in the Earth's Magnetosphere, Nauka, Moscow, 1982.

Sazhin, S. S. and M. Hayakawa, Magnetospheric chorus emissions: A review, Planet. Space Sci., 40, 681-697, 1992.

Singh, R. P., Whistler studies at low latitudes: a review, Ind. J. Rad. Spa. Phys., 22, 139-155, 1993.

Singh, Askok K., D. K. Singh, R. P. Patel, R. P. Singh, and A. K. Singh, Two types of ELF hiss observed at Varanasi, India, Ann. Geophysicae, 17, 1260-1267, 1999.

Singh, R., R. P. Patel, R. P. Singh, and Lalmani, An experimental study of hiss-triggered chorus emissions at low latitude, Earth Planets Space, 52, 37-40, 2000.

Singh, R. P., R. P. Patel, and D. K. Singh, Triggered emissions observed at Varanasi (India), Planet. Space Sci., 51, 495-503, 2003.

Singh, R. P. and R. P. Patel, Hiss triggered chorus emissions at Indian stations, J. Atmos. Solar Terr. Phys., 66, 1027-1033, 2004.

Singh, A. K. and K. Ronnmark, Generation Mechanism for VLF chorus emissions observed at low latitude ground station, Ann. Geophysicae, 24, 2067-2072, 2004.

Smirnova, N. A., Yu. P. Novikov, N. G. Kleimenova, and E. E. Titova, Some spectral peculiarities of VLF emissions registered on the Earth surface near the plasmapause projection, J. Atmos. Terr. Phys., 38, 1217 1976

Smirnova, N. A., A fine structure of the ground observed VLF chorus as an indicator of the wave particle interaction process in the magnetosphere, Planet. Space Sci., 32(4), 425-438, 1984.

Smith, A. J., M. P. Freeman, and G. D. Reeves, Post midnight VLF chorus events, a substorm signature observed at the ground near $L=4, J$. Geophys. Res., 101(A11), 24641-24653, 1996.

Storey, L. R. O., An Investigation of whistling atmospherics, Phil. Trans. Roy. Soc. London, A246, 113-141, 1953.

Titova, E. E., B. V. Kozelov, F. Jiricok, J. Smilauer, A. G. Demekhov, and V. Yu. Trakhtengerts, Varification of the backward wave oscillator model of VLF chorus generation using data from MAGION-5 satellite, Ann. Geophysicae, 21, 1, 2003.

Trakhtengerts, V. Y., Magnetosphere as an Alfven maser, Earth Universe, 4, 42, 1979.

Trakhtengerts, V. Y., Relaxation of plasmas with anisotropic velocity distribution, in Handbook of Plasma Physics, vol. 2, Basic Physics II, edited by A. A. Galeev and R. N. Sudan, pp. 519-552, Elsevier, New York, 1984

Trakhtengerts, V. Y., Magnetosphere cyclotron maser: Backward wave oscillator generation region, J. Geophys. Res., 100(A9), 17205-17210, 1995.

Trakhtengerts, V. Y., M. J. Rycroft, and A. G. Demekhov, Interrelation of noise-like and discrete ELF/VLF emissions generated by cyclotron interactions, J. Geophys. Res., 101(A6), 13293-13303, 1996.

Trakhtengerts, V. Y., A generation mechanism for chorus emission, Ann. Geophysicae, 17, 95-100, 1999.

Tsurutani, B. T. and E. J. Smith, Post midnight chorus: A substorm phenomena, J. Geophys. Res., 79, 118, 1974.

A. K. Singh (e-mail: abhay_s@rediffmail.com) and R. P. Singh 